\title{
SoLid: A compact neutrino detector for very short-baseline neutrino experiments
}

\author{
Leonidas N. Kalousis*广 \\ Vrije Universiteit Brussel \\ E-mail: leonidas.kalousisevub.ac.be
}

SoLid is currently one of the most compact and finely segmented neutrino detectors. Deployed near a compact and intense neutrino source, such as the Belgian BR2 research reactor, it is an ideal detector to search for sterile neutrino evidences.

A novel approach to measure reactor antineutrinos was developed, based on an innovative design of composite polyvynil-toluene (PVT) and ${ }^{6} \mathrm{LiF}: \mathrm{ZnS}$ scintillators. It consists of $5 \times 5 \times 5 \mathrm{~cm}^{3}$ cubes of PVT with ${ }^{6} \mathrm{LiF}: \mathrm{ZnS}$ sheets, read out by a network of wavelength shifting fibers and silicon photomultipliers (SiPMs). In this talk we will review the design, the operation characteristics, and the performance of a $288 \mathrm{~kg}$ full-size detector prototype that was deployed and operated at $\sim 5 \mathrm{~m}$ distance from the BR2 reactor core in 2015 .

The current design has been improved, with an installation foreseen in early 2017, and aiming to reach the 2.0 ton detector.

38th International Conference on High Energy Physics

3-10 August 2016

Chicago, USA

\footnotetext{
${ }^{*}$ Speaker.

${ }^{\dagger}$ On behalf of the SoLid collaboration.
} 


\section{Introduction: The SoLid experiment}

The recent observation of the Reactor Antineutrino Anomaly has revived the interest in shortbaseline experiments probing the disappearance of electron neutrinos and antineutrinos $[1,2]$. The SoLid experiment is a short-baseline reactor project, that aims to resolve the anomaly using a novel detector design. The SoLid detector will be installed at a distance of $\sim 5.5-10 \mathrm{~m}$ from the BR2 research reactor core at SCK•CEN (Mol, Belguim) and it will be able to scan the allowed parameter region through the detection of low-energy $\bar{v}_{e}$. The detector design allows for an efficient background reduction. This an important aspect for experiments at surface and near a nuclear reactor. SoLid is expected to start data taking with the Phase I detector in early 2017 and it will run in this configuration for several years.

BR2 is a nuclear reactor that is used for scientific research and the production of radioactive isotopes for medical applications. It is highly enriched in ${ }^{235} \mathrm{U}$ and it operates for approximately 150 days per year at a nominal thermal power of $\sim 60 \mathrm{MW}$. Due to the beta decays of neutron-rich fission fragments inside its core, a high-intensity flux of low-energy $\bar{v}_{e}$ is provided by BR2. Its compact dimensions (diameter of the core smaller than $\sim 0.5 \mathrm{~m}$ ) ensure that the neutrino oscillations, those predicted by the reactor anomaly, are not washed out by the spatial extension of the source. Furthermore, the SoLid site is ideal for performing such an experiment, since the background from the reactor is small compared to other locations.

SoLid will employ a long detector that covers a wide baseline range between $\sim 5.5$ to $10 \mathrm{~m}$. In this way, it will be able to probe neutrino oscillations as a function of the visible energy in the detector $\left(E_{v i s}\right)$ and distance from the reactor center $(L)$. Due to the close distance from the reactor, a high statistics $\bar{v}_{e}$ sample is expected in the SoLid detector. Nonetheless, for the purpose of the analysis a very good understanding of the detector systematics is necessary. In particular, the detector will need to have good energy and spatial resolutions.

The localization of $\bar{v}_{e}$ interactions in SoLid is provided by the detector granularity. This is a key feature of the experimental design and enhances dramatically the background reduction capabilities of the detector. Note that due to the close proximity with the nuclear reactor, and the fact that SoLid will be almost on surface (with only minimal overburden), a large amount of background events is anticipated. The detector segmentation together with the enhanced neutron tagging (owing to the addition of ${ }^{6} \mathrm{LiF}: \mathrm{ZnS}$ sheets) allows for a significant background reduction, as has been demonstrated with the prototype detector. Finally, we should also mention that SoLid will be also able to conduct a precise measurement of the ${ }^{235} \mathrm{U}$ flux and spectrum, and demonstrate the ability to perform reactor monitoring on surface.

\section{The SoLid detector design}

SoLid will employ a finely segmented detector made of $5 \times 5 \times 5 \mathrm{~cm}^{3}$ polyvinyl-toluene (PVT) plastic scintillator cubes. The cubes are optically separated through Tyvek wrappings and they are aggregated in $16 \times 16$ planes through the use of an aluminum frame. Squared wavelength shifting fibers are threaded through grooves on the cubes, in two orthogonal directions, allowing light to be detected by silicon photomultipliers (SiPMs) attached at the end of the fibers, Fig. 1. 


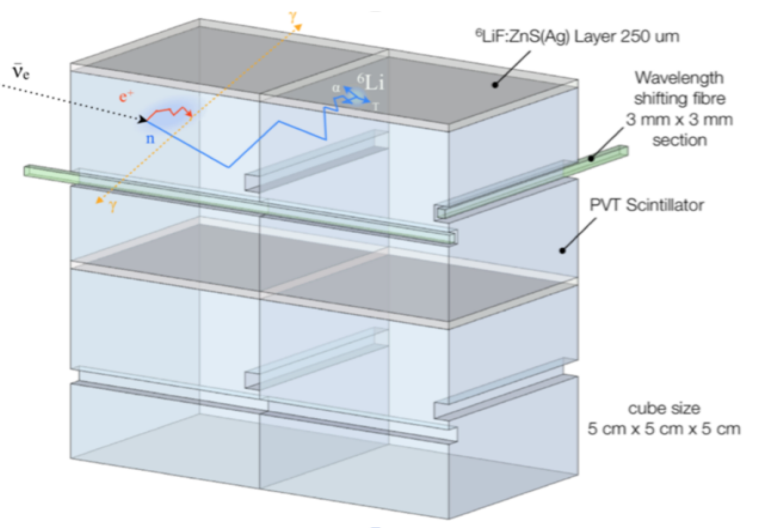

Figure 1: Simple schematic of the detector design and concept showcasing its main elements.
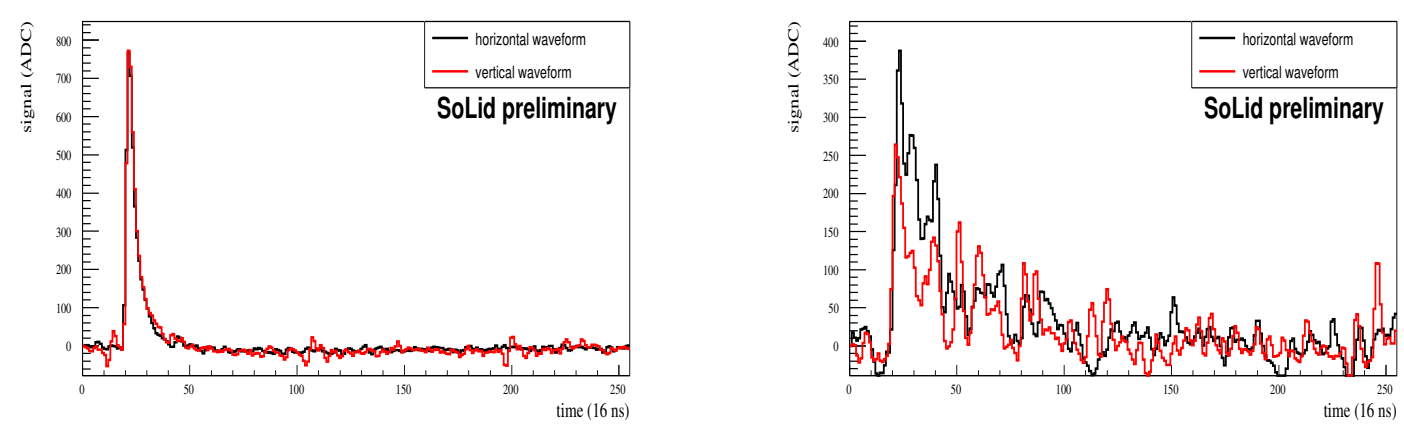

Figure 2: Signal waveforms for a prompt (left) and a delayed signal (right); the pulse amplitude in both plots is measured in ADC units. The qualitative differences between the pulses of the two events are evident.

With this design one can have precise spatial and calorimetric information of the neutrino interactions happening in the detector.

In the SoLid experiment, reactor antineutrinos are detected through inverse beta decay (IBD) interactions on free protons in PVT, $\bar{v}_{e}+p \rightarrow e^{+}+n$. This is a channel that has a rather high cross-section but energy threshold at $1.8 \mathrm{MeV}$. Following an IBD interaction, a prompt signal is first seen in the detector from the combined positron thermalization and annihilation. Subsequently, a secondary (delayed) signal is then observed from the capture of the neutron inside the detector, after the neutron thermalization. We should emphasize that these two signals are highly correlated in space (a few cubes distance) and time (time coincidence smaller than $500 \mu \mathrm{s}$ ).

In general, the neutron can capture on several materials. To tag the neutron, the SoLid technology is based on the use of ${ }^{6} \mathrm{LiF}: \mathrm{ZnS}$ sheets interleaved between the PVT cubes and the Tyvek covers, Fig. 1. The neutron capture cross-section on ${ }^{6} \mathrm{Li}$ is high and the delayed signal, $n+{ }^{6} \mathrm{Li} \rightarrow{ }^{3} \mathrm{He}+\alpha+4.78 \mathrm{MeV}$, can be identified with pulse shape discrimination (PSD) since the properties of the ${ }^{6} \mathrm{LiF}: \mathrm{ZnS}$ inorganic scintillator provide a very specific signal shape with respect to a signal in PVT. Fig. 2 shows the waveforms of a prompt (left) and a delayed (right) event. The delayed event has a long tail of peaks that is characteristic of the ${ }^{6} \mathrm{LiF}: \mathrm{ZnS}$ scintillator time-constants. This feature is very important in separating neutrons from electromagnetic signals (charged particles, gamma interactions, proton recoils) produced in the PVT cubes. 

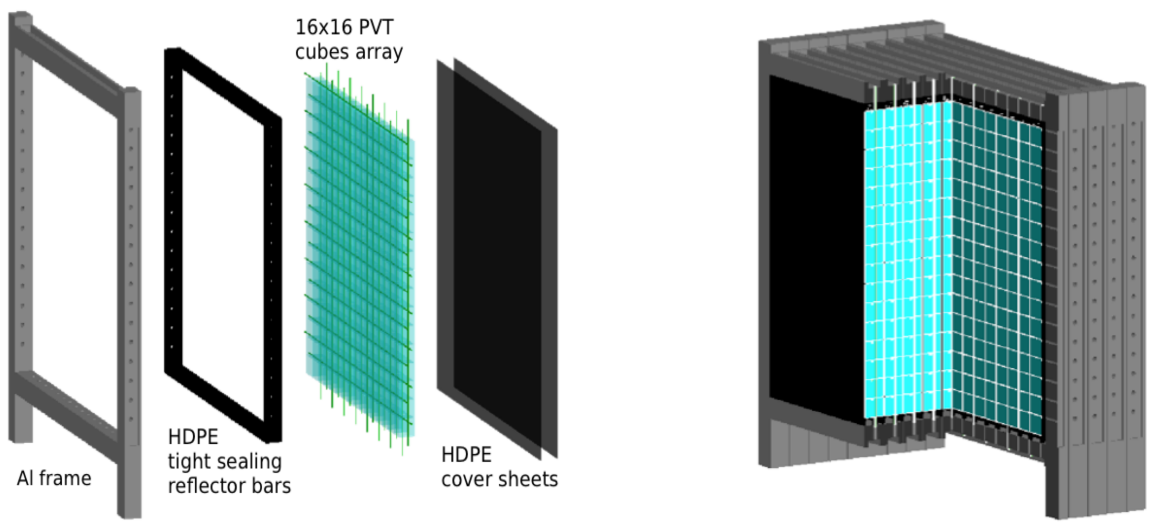

Figure 3: Schematics showing the basic elements of the SoLid detector planes (left) and a final schematic of the SM1 detector (right).

\section{SoLid detector prototypes}

The first instrument implementing the SoLid design was called NEMENIX and it was assembled in 2013. It was a small detector made out of 64 cubes and weighting $8 \mathrm{~kg}$. The main motivation for the construction of NEMENIX was the necessity to prove that one can build and operate such a detector, demonstrating the required neutron identification capabilities. NEMENIX took data at BR2 and showed good PSD between neutrons and electromagnetic signals. Moreover, it validated the ability to reconstruct prompt-delayed coincidences from reactor $\bar{v}_{e}$ and background.

The second detector prototype was called SM1 (2014-2015). It was a larger detector made out of 9 planes of $16 \times 16$ PVT cubes, offering an active mass of $288 \mathrm{~kg}$. It was mainly constructed to demonstrate the scalability of the detector concept to bigger volumes, test the production and schedule and finally demonstrate the power of the technology (PVT cube segmentation, use of ${ }^{6} \mathrm{LiF}: \mathrm{ZnS}$ screens for neutron capture, etc ...) to reduce efficiently the background. Furthermore, data from SM1 was used to develop the analysis tools and algorithms for event reconstruction and detector calibration.

In total, 2304 PVT cubes were machined for SM1. Each cube was cleaned and carefully weighted, so that its number of protons could be determined with an accuracy of better than $1 \%$. After that, the cubes together with the ${ }^{6} \mathrm{LiF}: \mathrm{ZnS}$ screens, were wrapped in Tyvek sheets and were grouped in planes of $16 \times 16$ through an aluminum frame. The square fibers were threaded and each plane was covered with high density polyethylene (HDPE) reflectors to reduce neutron dissipation. A schematic of the main elements that make up a plane are shown in Fig. 3 (left). Before installation each plane was tested and SM1 was built out of 9 detector planes, Fig. 3 (right).

SM1 took data at BR2 for 4 days of reactor on and approximately two months of reactor off, operating with a very good stability and performance. Additionally, a calibration campaign with radioactive sources (AmBe, ${ }^{252} \mathrm{Cf}$ and ${ }^{60} \mathrm{Co}$ ) was also performed. The analysis of these data sets showed an excellent discrimination between neutrons and electromagnetic signals (Fig. 4), while the calibration algorithms using cosmic muons, allowed to equalize channels at a level of better than $1 \%$. Furthermore, analyses with reactor off data validated (to first order) the Monte-Carlo background models used in SM1, and highlighted the background rejection capabilities of the 


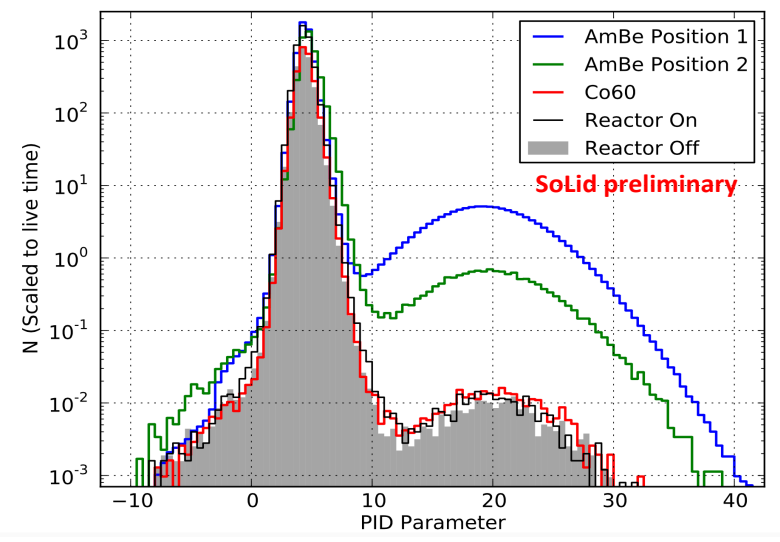

Figure 4: The distribution of the neutron identification parameter in SM1 for several data sets. We should mention that the neutron PSD in SM1, was performed using an total-to-amplitude algorithm exploiting information from both channels.

SoLid detector technology. For a more detailed discussion of the SM1 analyses and results, the interested reader might consult Ref. [3, 4].

\section{Phase I detector}

The SoLid Phase I detector has entered its construction phase and is expected to be completed and commissioned in early 2017. Phase I will be built on experience gained with SM1, but incorporating several improvements that will allow us to ensure our physics goals. It will be an even larger detector of 2.0 ton active mass and it will be an instrument capable to perform high precision physics measurements. Phase I will consist of 6 detector submodules of 10 planes of $16 \times 16$ cubes each, Fig. 5. Stated differently, it will be made of 60 planes totally and 15360 PVT cubes.

The design and mechanics of each plane are essentially the same as SM1, with the some minor modifications. The detector will be placed inside a container where the temperature will be controlled to a very precise level inside the container, ensuring low SiPM dark rates and a better detector stability. To improve the neutron detection efficiency in Phase I, every cube will be equipped with two ${ }^{6} \mathrm{LiF}: \mathrm{ZnS}$ sheets, increasing the capture efficiency on ${ }^{6} \mathrm{LiF}: \mathrm{ZnS}$ in about $30 \%$. Moreover, each cube will be read out by two optical fibers in each direction, as opposed to SM1 that employed one fiber per direction. This latter choice improves significantly the light collection (about $66 \%$ more light), increasing the energy resolution to $\sim 14 \% / \sqrt{E_{v i s}}$ in the Phase I detector. Note that in SM1 the energy resolution was $\sim 20 \% / \sqrt{E_{v i s}}$.

One of the major highlights of the Phase I detector is the so-called neutron trigger. More specifically, the event rate in Phase I is expected to be high. For this reason, the detector will be designed to have an online trigger, implemented at the data acquisition level. In contrast to SM1, the Phase I detector will trigger on neutrons and will readout only a part of the detector for a short time interval, $\sim 1 \mathrm{~ms}$, before and after this event. In this way most of the IBD interactions will be recorded, while the bulk of the background from single electromagnetic events can be omitted. 


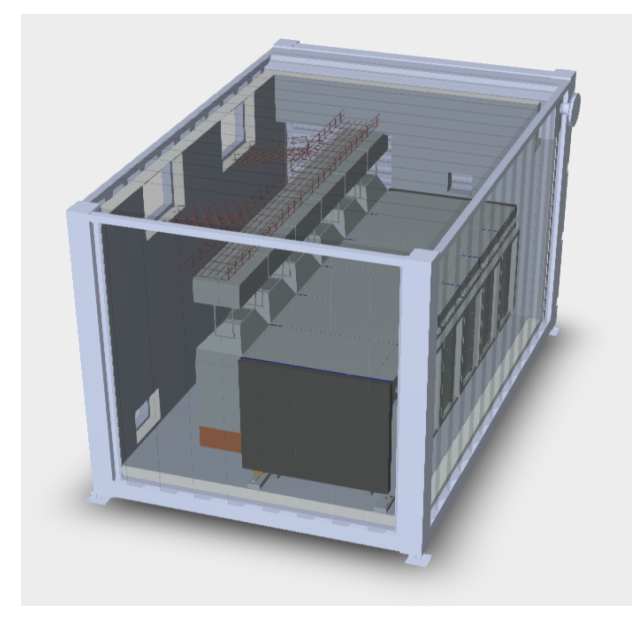

Figure 5: The SoLid Phase I detector.

Last, SoLid will employ two independent systems for the calibration of the Phase I detector: CALIPSO and CROSS. CALIPSO will be an off-site calibration system, using an articulated arm to calibrate detector planes with radioactive sources, prior to their installation. On the other hand, CROSS will be an on-site system, allowing to deploy sources and determine accurately the energy response and neutron detection efficiency of the detector in-situ.

\section{Conclusions}

The SoLid experiment is expected to perform a very precise search for the disappearance of low-energy $\bar{v}_{e}$ using a novel detector design. The operation of the SM1 detector prototype was met with great success, demonstrating excellent neutron discrimination, precise channel intercalibration with cosmics and very good background rejection. The SoLid collaboration has begun the construction of a 2.0 ton detector (Phase I), that is anticipated to start data taking in early 2017. According to sensitivity studies, SoLid will be able to scan most of the allowed parameter space within a few years of data taking [5].

\section{References}

[1] G. Mention et al., The Reactor Antineutrino Anomaly, Phys. Rev. D 83 (2011) 073006 [arXiv:1101.2755].

[2] K. N. Abazajian et al., Light Sterile Neutrinos: A White Paper, (2012) [arXiv: 1204.5379 ].

[3] I. Michiels (SoLid collaboration), Overview of the background reduction techniques applied in the SoLid experiment, these proceedings.

[4] D. Saunders (SoLid collaboration), SoLid: Search for Oscillations with a Lithium-6 Detector at the $S C K \cdot C E N B R 2$ reactor, these proceedings.

[5] L. N. Kalousis (SoLid collaboration), The SoLid experiment, proceedings of the 27th International Conference on Neutrino Physics and Astrophysics (Neutrino 2016), 4 - 9 July, 2016, London (UK). 